\title{
Current Status and Perception of Acne Among Chinese Adolescents: A Community-Based, Cross-Sectional Study
}

\author{
Jian Chun Hao", Yan Yu ${ }^{2}$, Shao Wei Cheng ${ }^{1}$, , Di Hui Lai ${ }^{1}$, Yu Fu ${ }^{1}$ \\ ${ }^{1}$ Department of Dermatology, Beijing Chuiyangliu Hospital, Beijing, China \\ ${ }^{2}$ Department of Traditional Chinese Medicine, Beijing Chuiyangliu Hospital, Beijing, China
}

Email address:

shorewave2008@139.com (Shao Wei Cheng)

\section{To cite this article:}

Jian Chun Hao, Yan Yu, Shao Wei Cheng, Di Hui Lai, Yu Fu. Current Status and Perception of Acne Among Chinese Adolescents: A Community-Based, Cross-Sectional Study. Science Journal of Public Health. Vol. 3, No. 6, 2015, pp. 836-841.

doi: $10.11648 /$ j.sjph.20150306.17

\begin{abstract}
Background: Acne is a common disease in adolescents, which may result in physical and psychological damage. Objective: This study was carried out to assess the current status and perceptions of adolescents toward acne in Beijing, China. Methods: A total of 858 students aged 12-18 years were asked to complete a self- administered questionnaire to determine their perception of acne prevalence, treatment, and satisfaction of treatment. Results: The prevalence of acne in the sample was $54.7 \%$. Adolescents obtained acne knowledge primarily from their parents (26.5\%). 56.9\% of adolescents were aware of acne; senior high school students were more aware of acne than junior high school students $(p<0.05)$. Only $35.2 \%$ of adolescents thought that they should go to the hospital for the treatment of acne; $16.8 \%$ actually did so, and $46.1 \%$ opted for no treatment. Junior high school students ignored treatment more often than did senior high school students $(p<0.05)$. Only $16.7 \%$ of students considered treatment to be effective. Conclusions: High acne prevalence existed in Chinese adolescents, but the number treated and efficacy of treatment were not satisfactory, which may be related to the poor perception of acne among this group.
\end{abstract}

Keywords: Acne, Adolescents, Prevalence, Perception

\section{Introduction}

Acne is often misunderstood by lay people, and even the medical community, to be merely part of growing up and a cosmetic problem, while increasing evidence has revealed that acne is not a common dermatologic disease; it can produce a significant psychosocial burden[1-6] and be comparable with such disabling maladies as asthma, epilepsy, diabetes, back pain, and arthritis[7].

Although the prevalence of acne varies among different populations in different studies[1,3,8,9], it is very common among teenagers[10,11]. Adolescence is an important and vulnerable period in most people's lives, as it is the time of transition from the dependence of childhood to the independence of adulthood, so this group is vulnerable both socially and psychologically. Since they are neither mature psychologically nor knowledgeable about acne, the potential harm of acne to adolescents is significant. Therefore, epidemiological studies on acne must befocused mainly on adolescents.

It is necessary that epidemiological studies of acne determine whether there is a need to educate both those affected and those health providers caring for them ${ }^{[8]}$. In this paper, we inquired about the current status of acne among adolescents in China, including prevalence, severity, treatment, efficacy of treatment, and psychological impact, and provided data to health providers to decide whether it is necessary to institute acne health education among adolescents.

Many studies have been designed to investigate the effect of acne on the quality of life among adolescents and the related risk factors, although few have been undertaken to research how this segment of the population perceives acne $[3,6,7,8,12-14]$. A recent worldwide study of patients with acne found that $50 \%$ reported that they knew little or nothing about the condition[15]. Lack of knowledge about acne may cause adolescents to delay treatment or to take inappropriate measures, resulting in physical or psychological damage. Therefore, it is necessary to understand the existing perceptions of adolescents about acne in order to improve their knowledge of this disease. This study should encourage the health educator to institute health education programs suitable for adolescents.

The purpose of this paper was to survey the current status and 
perceptions of acne among adolescents in Beijing, China, in order to promote the succedent health education regarding acne.

\section{Methodology}

\subsection{Subjects}

In this cross-sectional survey, 858 adolescents aged between 12 and 18 years were recruited. Of those, 837 (97.6\%) students responded to the questionnaire, including 413 boys (49.3\%) and 424 girls $(50.7 \%)$, of which 368 (44.0\%) were junior high school studentsand 469 (56.0\%) were senior high school students.

Communities were selected from random samples of schools in Beijing. To prevent loss of sample, random cluster sampling was adopted, taking a class as a unit. The study was granted ethical approval by the Ethics Committee of Beijing Chuiyangliu Hospital, and the students were informed about this survey before answering the questionnaire.

\subsection{Questionnaires}

The students were asked to complete a self-administered, multiple-choice questionnaire, using as a reference one employed in previous acne epidemiological studies ${ }^{[1,5,8,16]}$. Some questions were designed specifically for Chinese people. Instructionson correctly filling out the questionnaire were provided by the same researcher before the survey.

The questionnaire contained three parts: general information (gender, age, grade); perceptions about acne, including awareness of acne, harm to the skin, risk factors, opinions, preferred acne treatment, and source of knowledge; and current status of acne (prevalence, severity, treatment, efficacy of treatment, and psychological impact). Acne severity was represented by the forms of acne shown in distributed photos. Objective evaluation by a dermatologist present in the room was impossible because of school time limits.

\subsection{Statistical Analysis}

Statistical analysis was performed using SPSS 17.0 for Windows (SPSS Inc, Chicago, IL, USA). Relationships between boys and girls and senior and junior high school students were assessed via contingency tables using the $\chi^{2}$ test. A level of $p<0.05$ was considered statistically significant in all the analyses.

\section{Results}

\subsection{Current Status of Acne Among Adolescents}

The prevalence of acne in the sample was $54.7 \%$. There was no difference between boys and girls $(55.2 \%$ versus $54.2 \%, p>$ $0.05)$, while the difference was significant between senior and junior high school students $(62.5 \%$ versus $44.8 \%, p<0.05)$.

We investigated the expression of the three forms of acne (comedo, papule, pustule) and scar, representing the degree of acne from mild to severe. Comedo was the most common form of acne among adolescents (37.8\%). Boys tended to present papules more than did girls $(p<0.05)$, and there was no difference between senior and junior high school students in the presentation of comedo, papule, pustule, and scar $(p>0.05)$.

\subsection{Perceptions of Acne Among Adolescents}

Most adolescents (56.9\%) were aware of acne, but this awareness was not significant between boys and girls $(p>$ 0.05); however, senior high school students knew more about acne than junior high school students $(p<0.05)$.

Compared with boys and junior high school students, girls and senior high school students knew more about acne's harm to the skin ( $p<0.05$, Fig. 1). More boys and junior high school students either thought that acne is not harmful to the skin or had no idea of its potential harm than did girls and senior high school students ( $p<0.05$, Fig. 1).

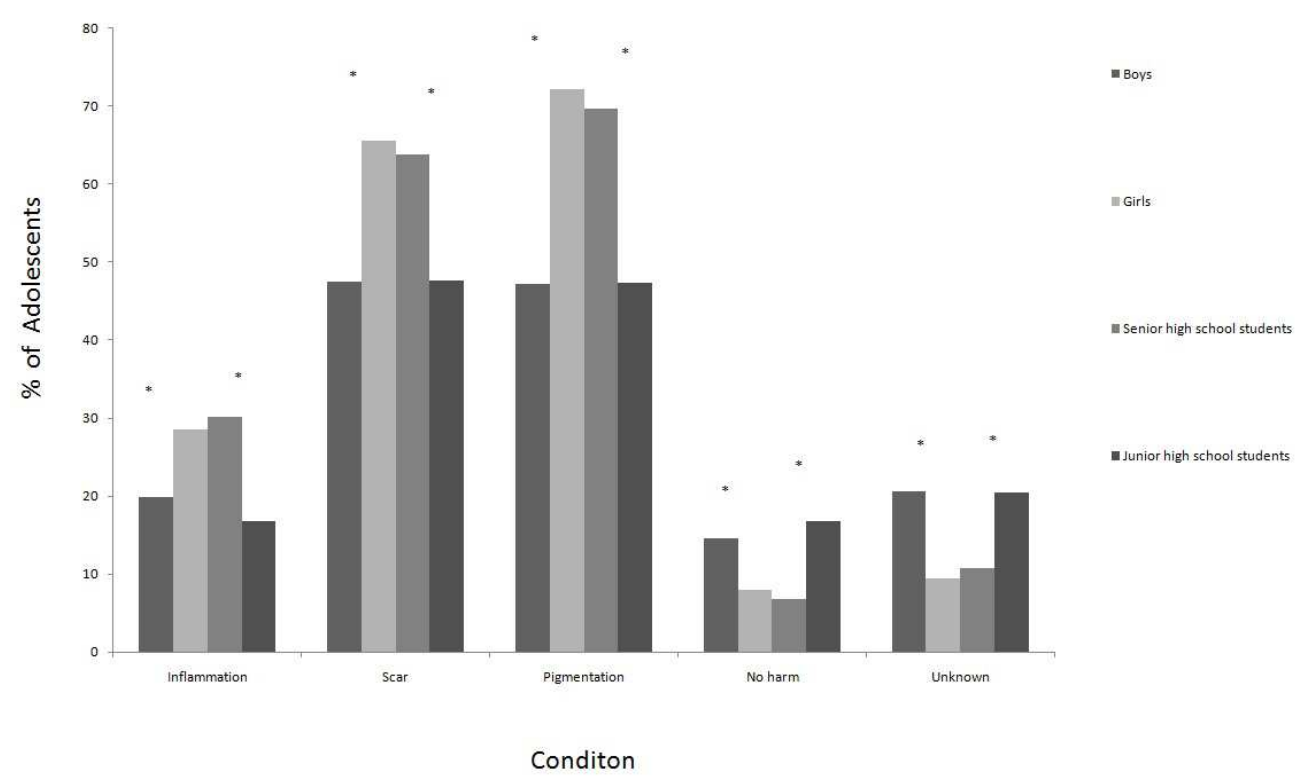

Fig. 1. Knowledge among adolescents of potential acne harm to skin. There was a difference between boys and girls and between senior and junior high school students $(* p<0.05)$. 
Fig. 2 illustrates the degree of knowledge of acne risk factors among the students. Girls and senior high school students knew more about these than did boys and junior high school students $(p<0.05)$.

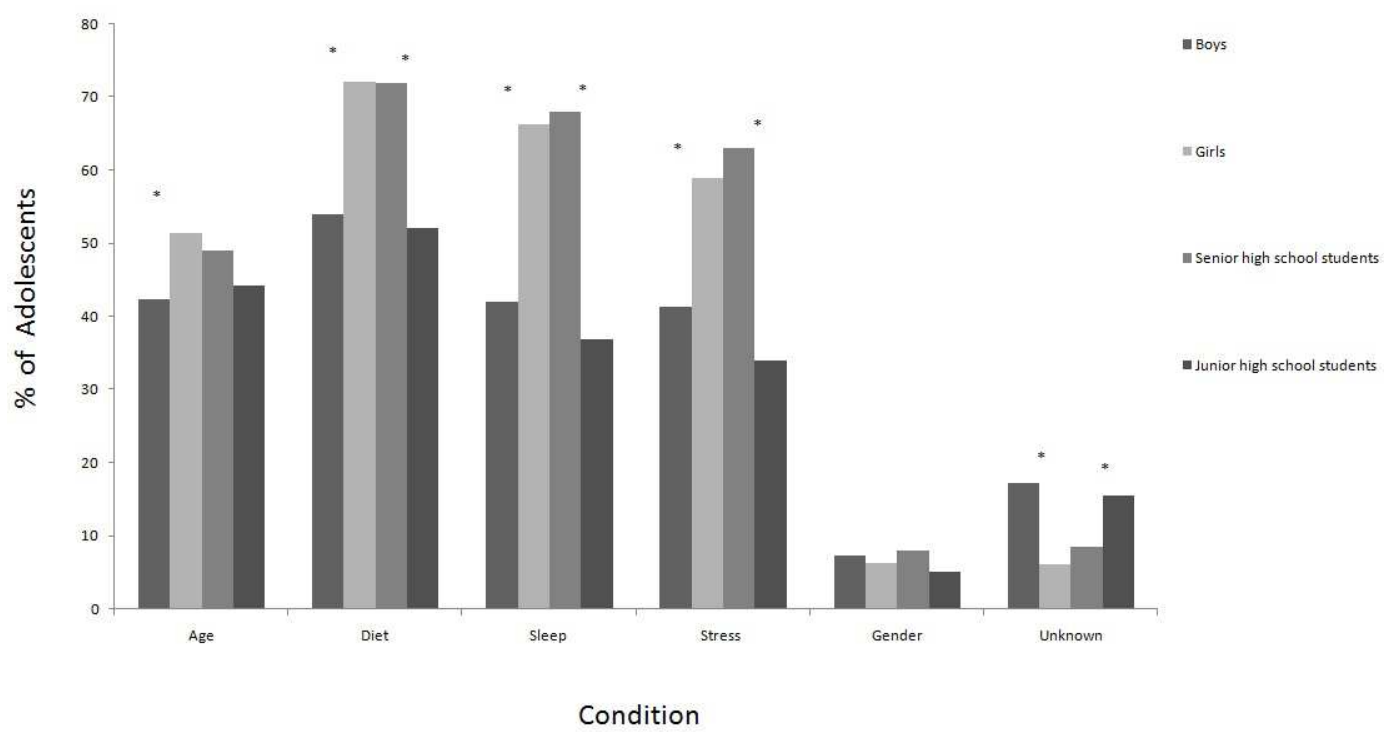

Fig. 2. Knowledge among adolescents about acne risk factors. There was a difference between boys and girls and senior and junior high school students $\left({ }^{*} p<\right.$ $0.05)$.

Adolescents obtained acne knowledge primarily from their parents $(26.5 \%)$, and then from their classmates (18.1\%) and the Internet $(17.6 \%)$. Girls preferred to get information about acne from classmates and the Internet compared with boys $(p<0.05)$.

As for the psychological effect of acne, $14.6 \%$ of teenagers reported effects such as distress and inferiority. Worrying about the impaired image caused by acne was the most notableimpact of acne on girls and both senior and junior high school students $(65.3 \%, 58.0 \%$, and $45.4 \%$, respectively), while there was no impact on most of the boys. More boys and junior high school students than girls and senior high school students believed acne had no influence on their life $(p<0.05)$, while more girls and senior high school students than boys and junior high school students were afraid of the scars impairing their image $(p<0.05)$. More junior high school students felt inferior than did senior high school students $(p<0.05)$.

\subsection{Treatment}

For treatment, $35.2 \%$ of adolescents thought they should go to hospital for acne, while $29.9 \%$ thought that there was no need for any treatment. There were statistical differences in treatment opinions between both genders and both grades ( $p<0.05$, Fig. 3 ).

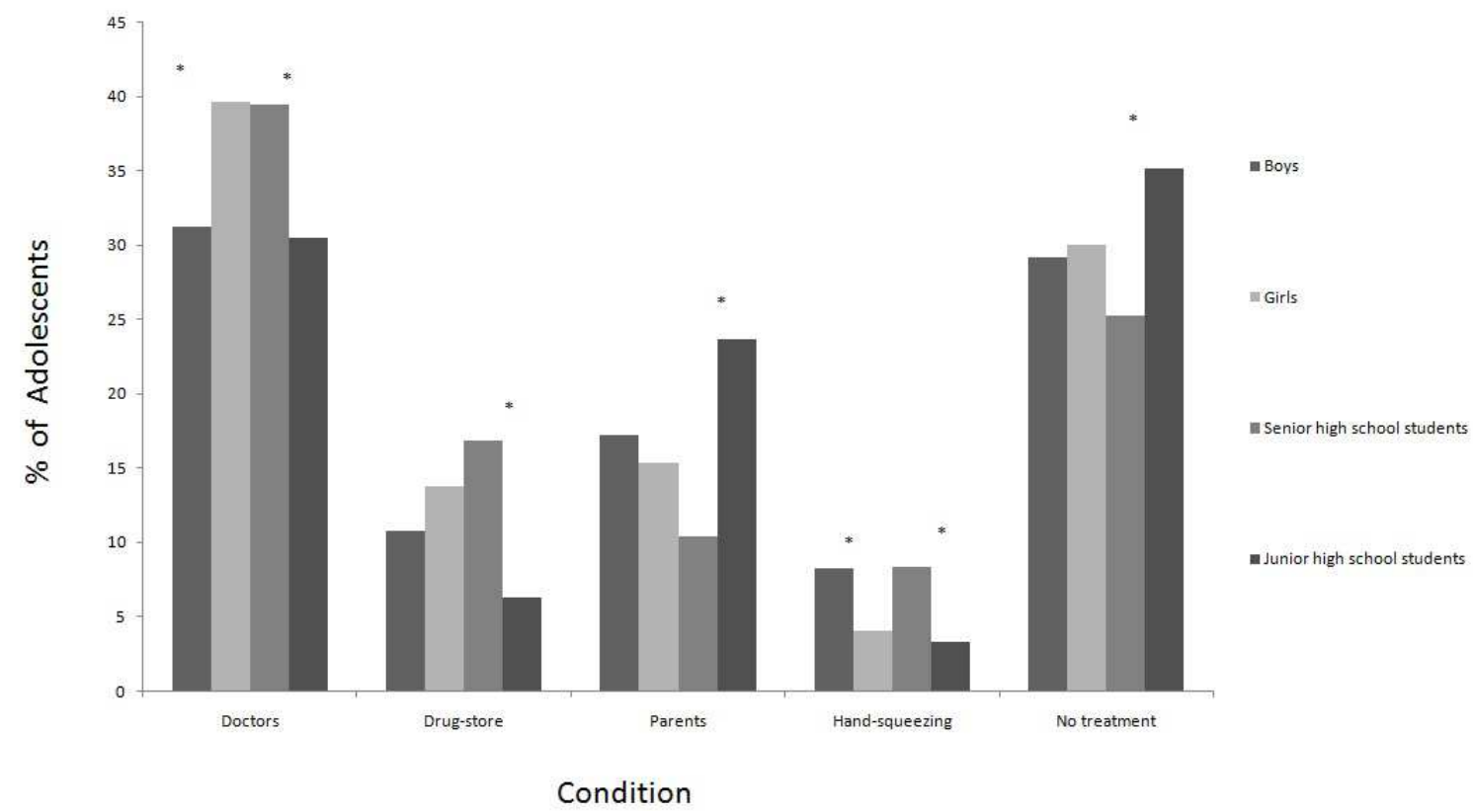

Fig. 3. Opinion of acne treatment among adolescents. There was a difference between boys and girls and senior and junior high school students (* $p<0.05)$. 
As for the intended measures, proper diet was the first choice checked by most adolescents (41.8\%), followed by topical medication $(26.4 \%)$, and oral medication $(8.2 \%)$. All the treatment preferences in this study were statistically different between both genders and grades $(p<0.05$, Fig. 4$)$.

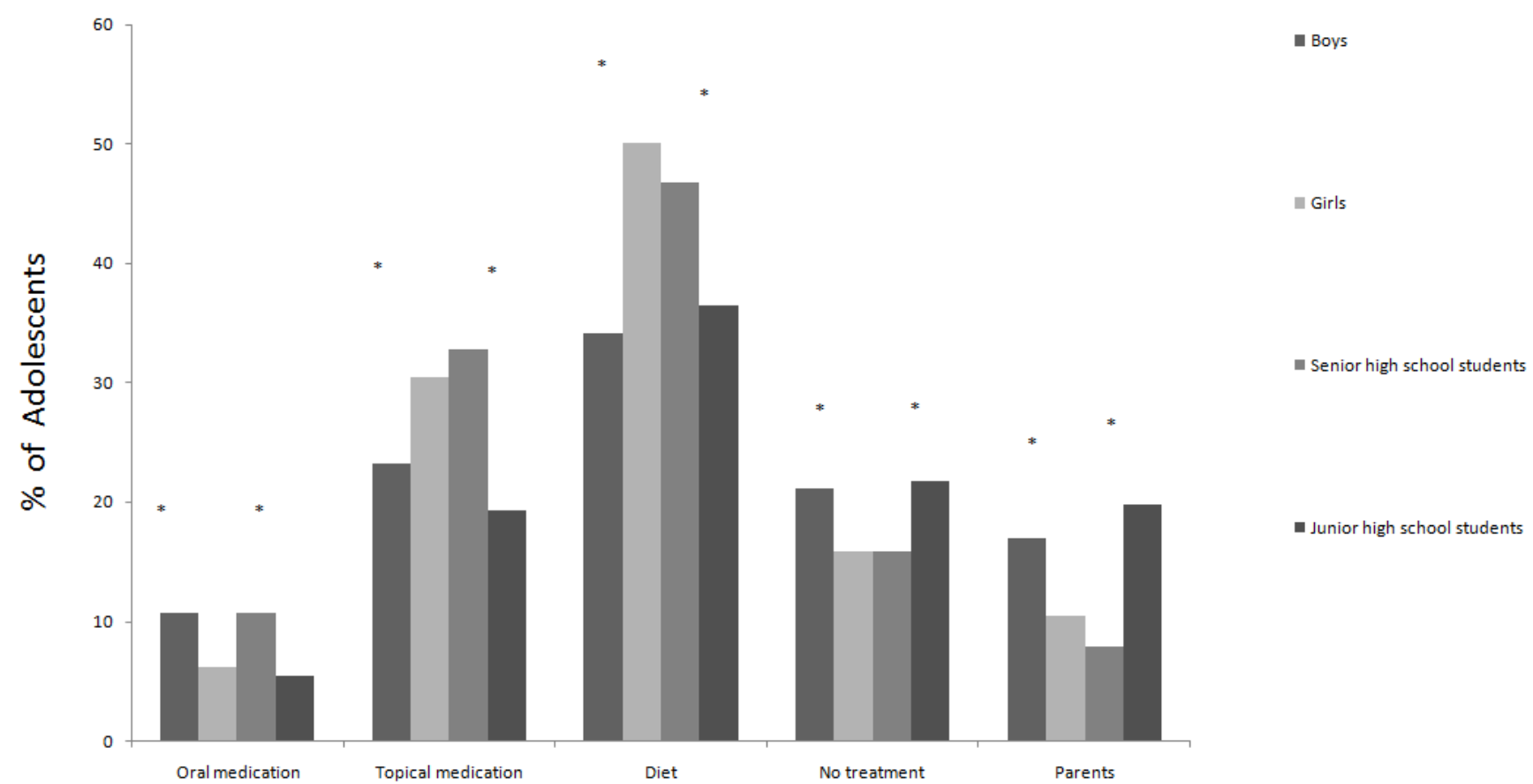

\section{Conditon}

Fig. 4. Preference of acne treatment among adolescents. There was a difference between boys and girls and senior and junior high school students ( $\left.{ }^{*} p<0.05\right)$.

Few $(16.8 \%)$ adolescents with acne went to hospital for treatment, $16.0 \%$ went to the drug store, and $24.3 \%$ were treated by their parents or themselves. Almost half of the adolescents (46.1\%) opted for no treatment, and junior high school students ignored treatment more often than did senior high school students $(53.4 \%$ and $42.4 \%$, respectively, $p<0.05)$. Girls got medication from the drug store by themselves more often than did boys $(p<0.05)$.

Only $16.7 \%$ of students considered treatment to be effective. More junior high school students thought treatment ineffective than did senior high school students $(p<0.05)$. Recurrence was reported by a small proportion of adolescents (15.2\%).

\section{Discussion}

Acne is a very common disorder in adolescents confirmed again by this study — having a prevalence of $54.7 \%$ in this representative sample of 12-to-18-year- olds. This is compatible with the prevalence of $59.5 \%$ reported in a recent Japanese study[3], but lower than that of other studies[17,18]. This difference might be related to ethnicand geographical differences. Self-reporting or diagnosing by a dermatologist in the discerning of acne may also explain some differences between studies. For example, Kubota et al[3], targeted a sample of Japanese teenagers, the same race as our study sample, and also used the same self-reporting method to evaluate the prevalence of acne, whereas Tan et al.[17] and Yahya [18] reported a prevalence of $88 \%$ and $90.7 \%$, respectively, in Singapore and Nigeria, which are located in tropical areas. Yahya [18] used an investigator rather than a self-reporting method.

We found that almost half the teenagers with acne did not undergo any treatment, and only $16.8 \%$ were treated by doctors, which was less than that reported by Shen et al[19]. and Law et al[20]. They preferred to buy medicine by themselves or squeeze the pustules by hand rather than seek help from doctors. These may indicate that the treatment of acne among the studied adolescents was delayed and that inappropriate methods were adopted widely. Similar results were found in some studies in which the majority of patients had no treatment for acne, thus treatment was postponed or the patient was self-treated[16,21], however, in contrast, in $\mathrm{Al}$ Robaee's study, most of his sample $(40.3 \%)$ sought medical advice within the first 3 months[18]. Otherwise, most adolescents were willing to regulate their diet to treat acne, but were strongly opposed to taking medication. The preference of acne treatment was different according to age and gender, which was useful information for subsequent health education.

This variability in treatment-seeking behavior may be related to underlying deficiencies in knowledge and beliefs about acne and the misunderstanding of acne treatment, 
assome studies reported[1,21], limited knowledge about acne is likely to impede seeking help. In the present study, among girls and older teenagers, the knowledge on acne, such as harm to the skin and risk factors, was better than that among boys and younger teenagers, which was supported by the study of Darwish[23]. This may explain why older teenagers and girls were more inclined to adopt some measures to deal with acne, even though these measures were not appropriate. These finding ssuggest that there is still room to improve teenagers' knowledge regarding risk factors and acne's harm to the skin. Correct therapy of acne should be imparted to adolescents through health education.

Furthermore, the lack of family doctors in China might be the cause of this low level of knowledge and inappropriate treatment of acne. Adolescents with acne should see a dermatologist in a large hospital, which might be intimidating. A heavy scholastic burden might be another reason for studentspreferring to buy OTC medicine rather than counseling with doctors.

An interesting finding in this study was that most teenagers believed acne should be treated by doctors, similar to Poli's study ${ }^{[24]}$, yet many chose no treatment and few sought help from the doctor. It is generally believed that it is easier to increase knowledge than to change behavior. Some studies have reported increases in knowledge without an intention of, or actual, improvement in behavior[25]. In our previous study, the same phenomenon was observed[26]. We called this phenomenon a "knowledge-behavior gap." In spite of the difficulty of changing intended or actual behavior[26,27], we are still trying to make acne treatment more accessible to students, for example, through regular acne examinations by a school physician. Since younger teenagers are more dependent on their parents for treatment, it is necessary that parents be well informed about acne.

We found that over half the adolescents experienced a psychological impact from acne, in agreement with other studies[1,3,27]. However, some were unaware of the harm of acne or believed no harm could ensue from acne, especially boys and younger teenagers, thus did not worry about it. We theorized that this was because of their low level of knowledge about acne rather than having the correct knowledge about acne just as an old Chinese saying says: "Knowing nothing, fearing nothing."

In our study, adolescents most frequently obtained general information on acne from parents, which is opposite to the findings of some studies[16,27,28], in which doctors were the primary source of acne information. The main source of acne knowledge among the adolescents was not doctors, but their parents, who are closest to adolescents. We concluded that the reasons might be the difficulty of seeing dermatologists and the lack of family doctors in China. Furthermore, dermatologists might fail to provide health education about acnebecause of heavy clinical loads, suggesting that it is necessary for parents to have a high level of acne knowledge. However, the question arises as to why teachers and school physicians - who are also close to the adolescents - are not the main source of acne knowledge. Two answers might be that (1) their heavy study burden allows teachers no time to provide health education for their students, and (2) acne knowledge is deficient among teachers and school physicians. Therefore, teenagers must obtain information about acne from parents, classmates, or the Internet. This finding suggests that we should provide more health education about acne in school, which will be the focus of our next work.

Limitations of this study include investigating only a localized study population and not doing face-to-face interviewing. The study was carried out in schools located in only one district of Beijing, thus the result does not represent the whole population in Beijing, let alone China. Of course, the study subjects represented a group of adolescents having a high level of knowledge and living in the most developed city of China. Therefore, we conclude that adolescents in China possess a low level of acne knowledge.

\section{Conclusion}

Our community-based study showed that the prevalence of acne among Chinese adolescents is high, which is related to age, but not gender. Most adolescents adopted no treatment or inappropriate treatment, thus the satisfaction with acne treatments was low, which may be related to poor acne knowledge. False perceptions about acne are prevalent among Chinese adolescents. It is urgent that acne knowledge among Chinese adolescents be improved through multiple sources.

\section{Acknowledgments}

The authors thank the subjects involved in this study for their cooperation and all investigators and dermatologists for their hard works and valuable contributions.

This study was supported by the Social Development Plan (Project No. SF1413) of Chaoyang District, Beijing, China.

\section{References}

[1] Wang P1, Wang H, Ding H, et al. Risk factors, psychological impacts and current treatments of acne in Shanghai area of China. J Dermatolog Treat 2015; 21:1-2.

[2] Dalgard F, Gieler U, Holm J, et al. Self-esteem and body satisfaction among late adolescentswith acne: results from a population survey. J Am Acad Dermatol 2008; 59:746-51.

[3] Kubota Y, Shirahige Y, Nakai K, et al. Community-based epidemiological studyof psychosocial effects of acne in Japanese adolescents. J Dermatol 2010; 37:617-22.

[4] Pruthi GK, Babu N. Physical and psychosocial impact of acne in adult females. Indian J Dermathol 2012; 57:26-9.

[5] Uslu G, Sendur N, Uslu M, et al. Acne: prevalence, perceptions and effects on psychological health among adolescents in Aydin, Turkey. J Eur Acad Dermatol Venereol 2008; 22:462-9.

[6] Do JE, Cho S-M, In S-I, er al. Psychosocial aspects of acne vulgaris: a community-based study with Korean adolescents. Annals of Dermatology 2009; 21:125-9. 
[7] Gieler U, Gieler T, Kupfer JP. Acne and quality of life impact and anagement. J Eur Acad Dermatol Venereol 2015; 29 Suppl 4:12-4.

[8] Ghodsi SZ, Orawa H, Zouboulis CC. Prevalence, severity, and severity risk factors of acne in high school pupils: a community-based study. J Invest Dermatol 2009; 129:2136-41.

[9] Collier CN, Harper JC, Cantrell WC, et al. The prevalence of acne in adults 20 years and older. $J$ Am Acad Dermatol 2008; 58:56-9.

[10] Kokandi A. Evaluation of acne quality of life and clinical severity inacne female adults. Dermatol Res Pract 2010; 2010. pii: 410809. doi: 10.1155/2010/410809. Epub 2010 Jul 27.

[11] Shaw L, Kennedy C. The treatment of acne. Paediatr Child Health 2007; 17:385-9.

[12] Jung JY, Yoon MY, Min SU, et al. The influence of dietary patterns on acne vulgaris in Koreans. Eur J Dermatol 2010; 20:768-72.

[13] Tasoula E, Gregoriou S, Chalikias J, et al. The impact of acne vulgaris on quality of life and psychic health in young adolescents in Greece. Results of a population survey. An Bras Dermatol 2012; 87:862-9.

[14] Safizadeh H, Shamsi-Meymandy S, Naeimi A. Quality of life in Iranian patients with acne. Dermatol Res Pract 2012; 2012:571516. doi: 10.1155/2012/571516. Epub 2012 Feb 15.

[15] Dréno B, Thiboutot D, Gollnick H, et al. Large-scale worldwide observational study of adherencewith acne therapy. Int J Dermatol 2010; 49:448-56.

[16] Tan JK, Vasey K, Fung KY. Beliefs and perceptions of patients with acne. J Am Acad Dermatol 2001; 44:439-45.

[17] Tan HH, Tan AW, Barkham T, et al. Community-based study of acne vulgaris in adolescents in Singapore. Br J Dermatol 2007; $157: 547-51$

[18] Yahya H. Acne vulgaris in Nigerian adolescents--prevalence, severity, beliefs, perceptions, and practices. Int J Dermatol 2009; 48:498-505.
[19] Shen Y, Wang T, Zhou C, et al. Prevalence of Acne Vulgaris in Chinese Adolescents and Adults:A Community-based Study of 17,345 Subjects in Six Cities. Acta Derm Venereol 2012; 92: $40-44$

[20] Law MP, Chuh AA, Lee A, Molinari N. Acne prevalenceand beyond: acne disability and its predictive factors among Chinese late adolescents in Hong Kong. Clin Exp Dermatol 2010; 35:16-21.

[21] Corey KC, Cheng CE, Irwin B, et al. Self-reported help-seeking behaviors and treatment choices of adolescents regarding acne. Pediatr Dermatol 2013; 30:36-41.

[22] Al Robaee AA. Prevalence, knowledge, beliefs and psychosocialimpact of acne in University students in central Saudi Arabia. Saudi Med J 2005; 26:1958-61.

[23] Darwish MA, Al-Rubaya AA. Knowledge, Beliefs, and Psychosocial Effect of Acne Vulgaris among Saudi Acne Patients. ISRN Dermatol 2013 Dec29; 2013:929340. doi: 10.1155/2013/929340. eCollection 2013.

[24] Poli F, Auffret N, Beylot C, et al. Acne as seen by adolescents: results of questionnaire study in 852 French individuals. Acta Derm Venereol 2011; 91:531-6.

[25] Buller DB, Buller MK, Beach B, Ertl G. Sunny days, healthy ways:evaluation of a skin cancer prevention curriculum for elementary school-aged children. J Am Acad Dermatol 1996; 35:911-22.

[26] Cheng S, Guan X, CaoM, et al. Randomized trial of the impact of a sun safety program on volunteers inoutdoor venues. Photodermatol Photoimmunol Photomed 2011; 27:75-80.

[27] Tallab TM. Beliefs, perceptions and psychological impact of acne vulgaris among patients in the Assir region of Saudi Arabia. West Afr J Med 2004; 23:85-7.

[28] Suh DH, Shin JW, Min SU et al. Treatment-seeking behaviors and related epidemiological features in Korean acne patients. $J$ Korean Med Sci 2008; 23:969-74. 